\title{
IMPACT OF SAR IMAGE RESOLUTION ON THE PERFORMANCE OF THE AMPLITUDE DISPERSION OPTIMIZATION FOR POLARIMETRIC PERSISTENT SCATTERER INTERFEROMETRY
}

\author{
Feng Zhao ${ }^{1}$, Jordi J. Mallorqui ${ }^{2}$, Juan M. Lopez-Sanchez ${ }^{3}$ \\ ${ }^{1}$ China University of Mining and Technology, Xuzhou, China \\ ${ }^{2}$ CommSensLab, Universitat Politècnica de Catalunya, Barcelona, Spain \\ ${ }^{3}$ IUII, Universitat d'Alacant, Alicante, Spain
}

\begin{abstract}
Polarimetric persistent scatterer interferometry (PolPSI) takes advantage of polarimetric optimization algorithms that enhance interferograms' phase quality by adequately combining the available polarization channels (e.g., $\mathrm{HH}, \mathrm{VV}, \mathrm{HV}$ and $\mathrm{VH})$ into an improved one. Amplitude dispersion $\left(D_{A}\right)$ is one of the commonly used phase quality metrics for this optimization. The resolution of the images is supposed to have an impact on the performance of $D_{A}$ based PolPSI in terms of both pixels density and quality. In this research, this impact is investigated. The results confirm that in all cases the ability of the $D_{A}$ to select high-quality pixels, i.e. Persistent Scatterers, decreases when the spatial resolution worsens because the loss of resolution increases the number of scatterers present in a resolution cell. However, for all employed resolutions (ranging from around $5 \mathrm{~m}$ to $20 \mathrm{~m}$ ) the polarimetric optimization improves the density and quality of PSs with respect to that of any single polarimetric channel. Moreover, this improvement is more noticeable, in relative terms, as the image resolution degrades.
\end{abstract}

Index Terms - Persistent scatterer interferometry (PSI), polarimetry, SAR image resolution, interferometric phase optimization

\section{INTRODUCTION}

Due to its high efficiency and millimetre accuracy of deformation monitoring, Persistent Scatterer Interferometry (PSI) with Synthetic Aperture Radar (SAR) data has been routinely used in terrain and infrastructures displacement detection and

This work has been supported by the Spanish Ministry of Economy, Industry and Competitiveness (MINECO), the State Research Agency (AEI) and the European Funds for Regional Development (EFRD) under projects TEC2017-85244-C2-1-P and TEC2017-85244-C2-2-P, in part by the National Natural Science Foundation of China (Grant 42004011, 41874044), and in part by the China Postdoctoral Science Foundation (Grant No. 2020M671646). It also has been supported by the CommSensLab, which is Unidad de Excelencia Maria de Maeztu MDM-2016-0600 financed by the AEI, Spain. Radarsat-2 data were provided by MDA in the framework of the scientific project SOAR-EU 6779. monitoring. Despite its successful performance in monitoring ground deformations related with subsidence, landslides and other geological environmental hazards, in some areas conventional PSI techniques may suffer with the lack of acceptable densities of coherent scatterers.

To improve the performance of conventional PSI techniques in these cases, two categories of algorithms have been proposed. On the one hand, SqueeSAR and similar techniques incorporate the use of distributed scatterers (DSs). On the other hand, according to the Polarimetric SAR Interferometry (PolInSAR) theory [1], the observed targets respond differently to different polarizations. Hence, by searching for the optimum scattering responses for each pixel its interferometric phase quality can be largely improved. Thus, the Polarimetric Persistent Scatterers Interferometry SAR (PolPSI) has been proposed [2-5].

PolPSI techniques improve the phase quality of SAR pixels by searching for their optimal scattering response from the point of view of an optimization criterion. The more the scattering mechanisms present within a resolution cell, the more difficult to find the optimum response. As the resolution worsens, SAR pixels are more likely to contain more diverse scattering mechanisms, thus it can be anticipated that pixels' resolution may have significant influence on the performance of PolPSI optimization techniques. However, to the best of the authors' knowledge, this issue has not been investigated in detail. In this paper, the impact of SAR image resolution on the PolPSI optimization with deterministic scatterers using the dispersion of amplitude, $D_{A}$, pixels selection criterion is investigated. The study has been done based on five quadpol SAR data sets. From the original one, with a resolution around $5 \mathrm{~m} \times 5 \mathrm{~m}$, four others have been generated with degraded resolutions up to $20 \mathrm{~m} \times 20 \mathrm{~m}$.

\section{POLARIMETRIC PERSISTENT SCATTERER INTERFEROMETRY WITH $D_{A}$ OPTIMIZATION}

The PolInSAR vector can be defined as

$$
\boldsymbol{K}=\left[\boldsymbol{k}_{1}, \boldsymbol{k}_{2}\right]^{T}
$$


where $\boldsymbol{k}_{1}$ and $\boldsymbol{k}_{2}$ are the two scattering vectors, defined by the set of polarimetric channels measured, from the master and slave PolSAR images that form the interferometric pair. Each polarimetric channel would generate a different interferogram. A single interferogram can be synthesized based on $\boldsymbol{K}$ with two normalized complex projection vectors $\boldsymbol{\omega}_{1}$ and $\boldsymbol{\omega}_{2}$, which are usually regarded as Scattering Mechanisms (SM), that combine for each image all available polarimetric channels into a single one [1]. The two original scattering vectors $\boldsymbol{k}_{1}$ and $\boldsymbol{k}_{2}$ are projected onto the resulting SM with,

$$
\mu_{i}=\boldsymbol{\omega}_{\boldsymbol{i}}^{\dagger} \cdot \boldsymbol{k}_{i}, \quad i=1,2
$$

where $\dagger$ refers to the conjugate transpose, $\mu_{1}$ and $\mu_{2}$ are two scalar scattering coefficients, analogous to single-polarization SAR images [1]. To avoid introducing artificial changes along time in the phase centers of the scatterers in PolPSI applications, $\omega_{1}$ and $\omega_{2}$ are forced to be identical, i.e. a single projection vector $\boldsymbol{\omega}$ is employed for all interferograms of the dataset [4].

The expression for the vector interferogram can be obtained as [1]

$$
\operatorname{Intf}=\mu_{1} \cdot \mu_{2}^{*}
$$

where ${ }^{*}$ is the complex conjugate. The pixel phase quality criterion $D_{A}$ can be then expressed as

$$
D_{A}=\frac{\sigma_{A}}{m_{A}}=\frac{1}{\overline{\left|\boldsymbol{\omega}^{\dagger} \boldsymbol{k}\right|}} \sqrt{\frac{1}{N} \sum_{i=1}^{N}\left(\left|\boldsymbol{\omega}^{\dagger} \boldsymbol{k}_{i}\right|-\overline{\left|\boldsymbol{\omega}^{\dagger} \boldsymbol{k}\right|}\right)^{2}}
$$

with

$$
\overline{\left|\boldsymbol{\omega}^{\dagger} \boldsymbol{k}\right|}=\frac{1}{N} \sum_{i=1}^{N}\left|\boldsymbol{\omega}^{\dagger} \boldsymbol{k}_{i}\right|
$$

where $\sigma_{A}$ and $m_{A}$ are the standard deviation and mean of the images' amplitudes, $N$ is the number of images and the over line indicates the empirical mean value [4].

The polarimetric optimization finds for each pixel the $\boldsymbol{\omega}$ that minimizes its $D_{A}$. An exhaustive search of the polarimetric space, also known as ESPO method [4], has been used as it provides the best performance in interferometric phase optimization among all possible methods. After the polarimetric optimization, through (2), the optimized SAR images are obtained. Then, the PSI processing can be carried out using the same PSI algorithms as for the single-pol case.

\section{DATA SET}

The data set is formed by 31 stripmap quad-pol Radarsat-2 images with a resolution of around $5 \mathrm{~m} \times 5 \mathrm{~m}$ that were acquired from May 2010 to July 2012 over the city of Barcelona. Additional data sets with coarser resolutions are generated from this original set of images. In order to simulate the acquisitions from a sensor with lower resolution, a down-sampling has been carried out in the spectral domain.
The original spectrum, in any of the two dimensions, is unweighted to obtain an almost flat spectrum. Depending on the resolution reduction factor, which sets the new bandwidth, a rectangular window is applied to eliminate the frequencies beyond the ones fixed by the new sampling frequency. Finally, the spectrum is weighted with the original window scaled to the new bandwidth. In practice, the last two steps are computed at the same time. In the azimuth dimension there are two extra steps. At the beginning, the image is base-banded as a function of the range-dependant Doppler centroid of the acquisition. After the resolution reduction, the final image is re-banded to its original Doppler centroid. Image parameters and orbital information are updated according to the new image bandwidths and sampling. Each new set of images is processed as if the images came for a real sensor. Therefore, a total of 4 new quad-pol data sets have been generated with resolutions around $7.5 \mathrm{~m} \times 7.5 \mathrm{~m}, 10 \mathrm{~m} \times 10 \mathrm{~m}$, $15 \mathrm{~m} \times 15 \mathrm{~m}$ and $20 \mathrm{~m} \times 20 \mathrm{~m}$, respectively.

\section{RESULTS AND ANALYSIS}

\subsection{Results of the $D_{A}$ Optimization}

The cumulative histograms of $D_{A}$ for $\mathrm{HH}$ and for the optimizations provided with different combinations of input polarimetric channels are depicted in Fig. 1 at all the spatial resolutions considered. It is worth to mention that the results corresponding to the $\mathrm{VV}+\mathrm{HV}$ polarimetric combination are very similar to those of the $\mathrm{HH}+\mathrm{HV}$ case, thus they are not presented. As Fig. 1 shows, for all resolutions the percentage of pixels with $D_{A}$ below 0.4 are around $10 \%, 40 \%$ and $80 \%$, for the $\mathrm{HH}$ channel [see Fig. 1(a)], the optimized dual-pol cases [see Fig. 1(c) and (g)], and the optimized quad-pol case [see Fig. 1(e)], respectively. This indicates that the polarimetric optimization improves the overall phase quality for all input data sets and for all resolutions. As expected, the quadpol data set always performs much better than the dual-pol ones.

For all input polarimetric combinations, the percentage of highest quality pixels (i.e., pixels with $D_{A}$ less than 0.25 ) decreases as the images' resolution worsens. However, and surprisingly, the relative improvement in the pixels quality with respect to the $\mathrm{HH}$ channel increases as the resolution worsens. For instance, the improvement achieved with the quadpol data with respect to $\mathrm{HH}$ is around $641 \%, 1,144 \%$ and $1,293 \%$ for the data sets with resolutions of $5 \mathrm{~m}, 15 \mathrm{~m}$ and $20 \mathrm{~m}$, respectively.

The impact of SAR images' resolution on the performance of the $D_{A}$-based polarimetric optimization is the result of the combination of two main factors. In first place, with the enlargement of the spatial resolution, each resolution cell has more chances to contain a higher number of significant scatterers with diverse scattering mechanisms, and therefore a particular pixel is more unlikely to behave as a persistent 

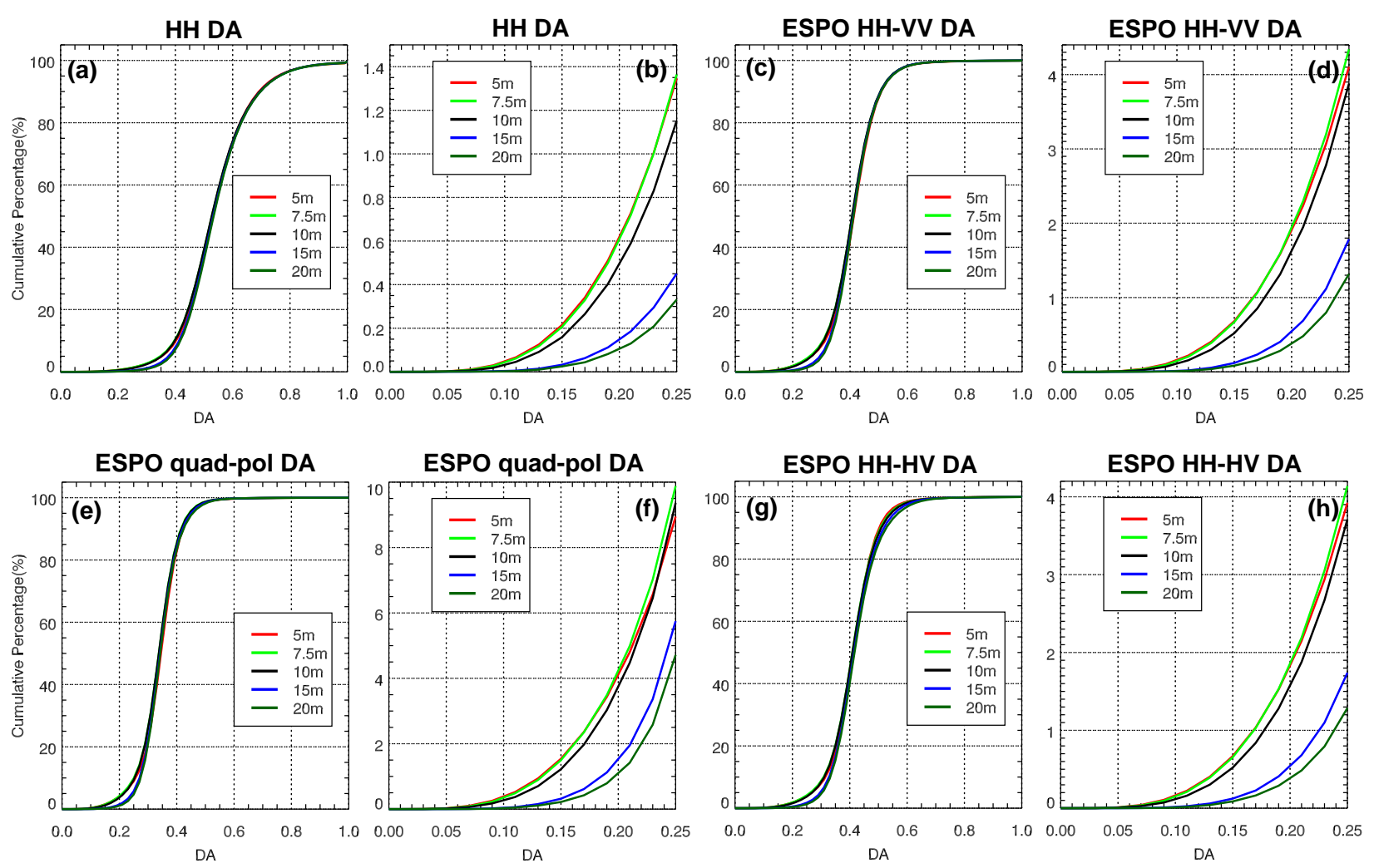

Fig. 1. (a), (c), (e) and (g) shows the histogram of $D_{A}$ cumulative percentage derived by HH, ESPO HH+VV, ESPO quad-pol and ESPO HH+HV polarimetric channels, respectively. (b), (d), (f) and (h) are a detailed zoom of (a), (c), (e) and (g) for $D_{A}$ values from 0 to 0.25 . It should be noted that the vertical axis is different for each zoomed plot (i.e., (b), (d), (f) and (h)).

scatterer. The significant decrease of the percentage of pixels with $D_{A}<0.25$ (see Fig. 1) in the single channel case when the resolution worsens from 10 to $20 \mathrm{~m}$ proves this point. In second place, the presence of the diverse scattering mechanisms in a pixel increases the chances of optimizing their amplitude dispersion with polarimetric optimization tools. In other words, in the image there will be a higher percentage of pixels with mixed scattering mechanisms that can be benefited of the polarimetric optimization.

\subsection{Deformation Estimation Result}

Pixels with $D_{A}$ less than 0.25 are selected as high quality pixels for the PSI processing for all polarimetric combinations and for the different resolutions considered. Ground deformation estimation is carried out with SUBSIDENCE-GUI, the PSI processing chain of Universitat Politècnica de Catalunya (UPC) that implements the Coherent Pixels Technique.

The estimated ground deformation over Barcelona retrieved from the HH channel and from the optimized quadpol data with three different spatial resolutions are shown in Fig. 2. Despite the differences in PS pixel density, the ground deformation patterns obtained from the different approaches and data sets are in good agreement with each other. In addition, these results are also in good accordance with those of the previous studies [4], which validates the correctness of the deformation maps.

The number of PSs in the estimated ground deformation results with all the data sets are listed in Table 1. The detailed results support previous conclusions. The highest densities of pixels are obtained with high-resolution optimized quad-pol data, but in all cases the optimization is able to increase the number of selected pixels. In addition, the highest improvements in percentage relative to the HH channel are achieved with the lowest resolution data.

\section{CONCLUSION}

The impact of the spatial resolution of SAR images on the polarimetric optimization of $D_{A}$ for PSI processing has been investigated. The tests carried out have allowed to obtain the following main conclusions: (1) The $D_{A}$-based polarimetric optimization improves the density of PS pixels with respect to a single-pol reference channel for any input PolSAR data set, either dual- or quad-pol, and with any resolution ranging from $5 \mathrm{~m}$ to $20 \mathrm{~m}$. (2) The improvement in density of PSs 
Table 1. Number of PSs obtained by using $D_{A}$ based approaches with different polarimetric combinations over the five different resolution data sets.

\begin{tabular}{l|c|c|c|c|c}
\hline \hline \multicolumn{1}{c|}{ Pol. } & HH & Quad-pol & HH+VV & HH+HV & VV+HV \\
Res. & $78454(0 \%)$ & $499028(536 \%)$ & $239363(205 \%)$ & $229955(193 \%)$ & $231322(195 \%)$ \\
$\mathbf{5 m}$ & $34841(0 \%)$ & $236578(579 \%)$ & $110781(218 \%)$ & $106404(205 \%)$ & $104834(201 \%)$ \\
$\mathbf{7 . 5 m}$ & $16315(0 \%)$ & $123544(657 \%)$ & $54631(239 \%)$ & $52818(224 \%)$ & $51194(214 \%)$ \\
$\mathbf{1 0 m}$ & $2704(0 \%)$ & $30015(1010 \%)$ & $10392(284 \%)$ & $10391(284 \%)$ & $9817(263 \%)$ \\
$\mathbf{1 5 m}$ & $1110(0 \%)$ & $13068(1077 \%)$ & $4248(283 \%)$ & $4260(284 \%)$ & $4006(261 \%)$ \\
$\mathbf{2 0 m}$ & \hline
\end{tabular}

'Res.' and 'Pol.' are the abbreviations of 'Resolution' and 'Polarimetric channel', respectively. "N (j\%)" in the table represent the final number of PSs $(\mathrm{N})$ and its increase $(\mathrm{j} \%)$ w.r.t. that of the single $\mathrm{HH}$ polarimetric approach.
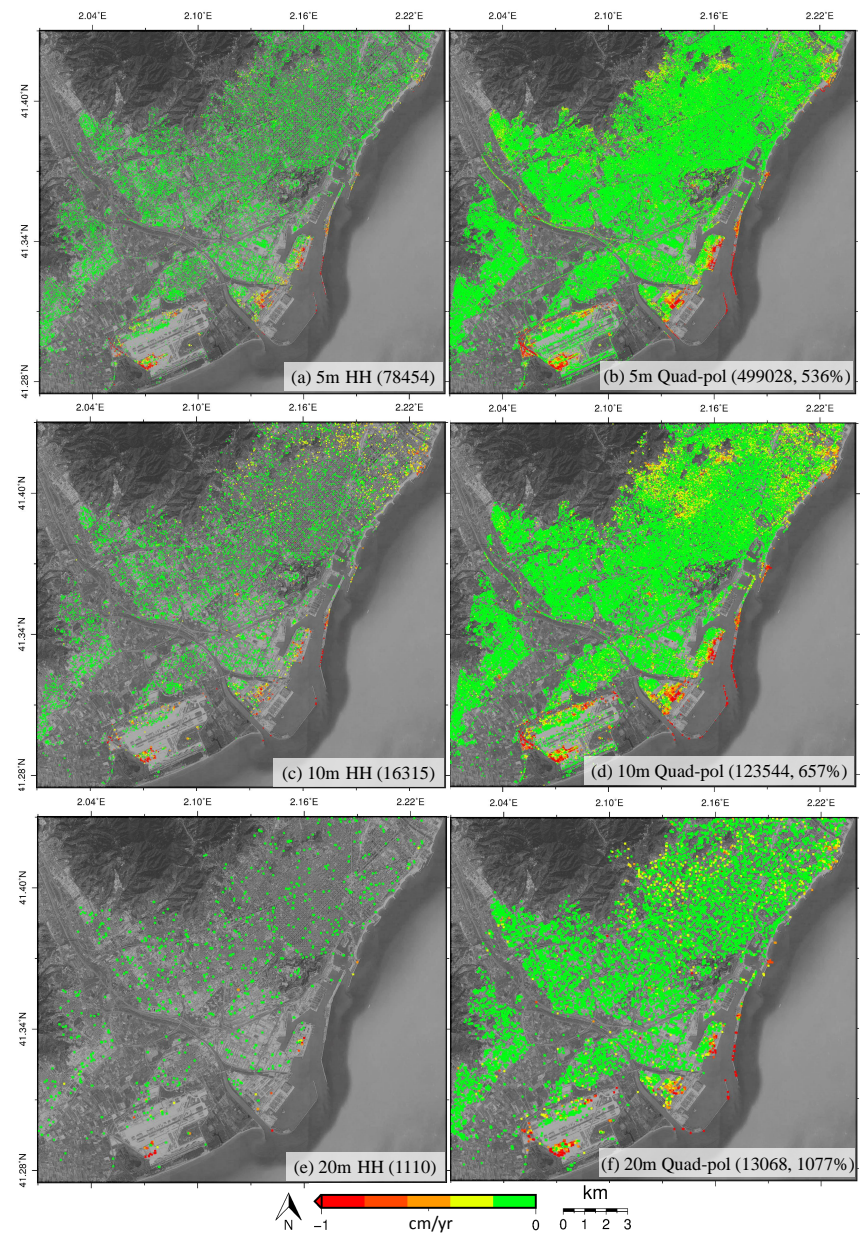

Fig. 2. Ground deformation velocity estimated with $5 \mathrm{~m}$ (top), $10 \mathrm{~m}$ (middle) and $20 \mathrm{~m}$ (bottom) resolution data sets, respectively. (a), (c), (e) are the results derived from the $\mathrm{HH}$ channel, and (b), (d), (f) from the optimized quad-pol data. The number in brackets represents the final number of PS pixels obtained by each approach, and the improvement percentage is obtained by taking the $\mathrm{HH}$ channel as a reference for each resolution case. is more significant in percentage with respect the reference channel as the image resolution worsens. (3) From this study, it can be also concluded that it is worth processing the two channels (i.e, VV and VH) of the Sentinel-1 data by applying the polarimetric optimization with $D_{A}$ for PSI applications.

\section{REFERENCES}

[1] Shane R Cloude and Konstantinos P Papathanassiou, "Polarimetric SAR interferometry," IEEE Transactions on Geoscience and Remote Sensing, vol. 36, no. 5, pp. 1551-1565, 1998.

[2] Luca Pipia, Xavier Fabregas, Albert Aguasca, Carlos Lopez-Martinez, Sergi Duque, Jordi J Mallorqui, and Jordi Marturia, "Polarimetric differential SAR interferometry: First results with ground-based measurements," IEEE Geoscience and Remote Sensing Letters, vol. 6, no. 1, pp. 167-171, 2009.

[3] Victor D Navarro-Sanchez, Juan M Lopez-Sanchez, and Fernando Vicente-Guijalba, "A contribution of polarimetry to satellite differential SAR interferometry: Increasing the number of pixel candidates," IEEE Geoscience and Remote Sensing Letters, vol. 7, no. 2, pp. 276-280, 2010.

[4] Ruben Iglesias, Dani Monells, Xavier Fabregas, Jordi J Mallorqui, Albert Aguasca, and Carlos Lopez-Martinez, "Phase quality optimization in polarimetric differential SAR interferometry," IEEE Transactions on Geoscience and Remote Sensing, vol. 52, no. 5, pp. 2875-2888, 2014.

[5] Feng Zhao and Jordi J. Mallorqui, "Coherency Matrix Decomposition-Based Polarimetric Persistent Scatterer Interferometry," IEEE Transactions on Geoscience and Remote Sensing, vol. 57, no. 10, pp. 7819-7831, 2019. 